\title{
Prescaled Integer Division
}

\author{
David W. Matula, Alex Fit-Florea \\ Department of Computer Science and Engineering \\ Southern Methodist University \\ \{matula,alex\}@engr.smu.edu
}

\begin{abstract}
We describe a high radix integer division algorithm where the divisor is prescaled and the quotient is postscaled without modifying the dividend to obtain an identity $N=Q^{*} \times D+R^{*}$ with the quotient $Q^{*}$ differing from the desired integer quotient $Q$ only in its lowest order high radix digit. Here the "oversized" partial remainder $R^{*}$ is bounded by the scaled divisor with at most one additional high radix digit selection needed to reduce the partial remainder and augment the quotient to obtain the desired integer division result $N=Q \times D+R$ with $0 \leq R \leq D-1$.

We present a high radix multiplicative version of this algorithm where a $k \times p$ digit base $\beta$ rectangular aspect ratio multiplier allows quotient digit selection in radix $\beta^{k-1}$ with a cost of only one $k \times p$ digit multiply per high radix digit, plus the fixed pre-and post-scaling operation costs. We also present a Booth radix 4 additive version of this algorithm where appropriately compressed representation of the partial remainder with Booth digits $\{-2,-1,0,1,2\}$ allows successive quotient digit selection from the leading partial remainder digit without the iterative table lookups required in SRT division.
\end{abstract}

\section{Introduction}

Digit serial division algorithms generating quotient digits in a higher radix in most significant digit first order are well known. The SRT division procedure characterized by Atkins in [1] iteratively employs table lookup for determining the next quotient digit and an adder to update the partial remainder. Using a shortby-long rectangular aspect ratio multiplier the digit serial division procedure may be implemented in a much higher radix using multiplication of the partial remainder by a short reciprocal of the divisor with rounding to determine the next quotient digit. Updating the remainder is then accomplished employing another short-bylong multiply. See [3] and [5] for an extensive list of references on these methods.

Prescaled division is an enhanced version of digit se- rial division introduced by Svoboda in [9] that has been followed with a rich literature noted in [5]. The process of prescaled division scales both the numerator and denominator by a predetermined short reciprocal of the divisor. Division of the scaled numerator by the scaled divisor yields the identical quotient to any precision with a remainder that is implicitly also scaled by the short reciprocal. The efficiency advantage of prescaled division does not apply to integer division as the necessary additional work to effectively "descale" the scaled remainder costs as-much-as or more than the savings gained.

We present an alternative methodology for prescaled integer division of a dividend $N$ by a divisor $D$ that provides both the quotient $Q$ and remainder $R$, achieving almost the same efficiency as that of a straightforward prescaling procedure providing the same quotient with a scaled remainder. Our algorithm involves divisor prescaling and quotient postscaling. That is, the (unscaled) dividend is divided by the scaled divisor resulting in a "reduced" integer quotient and an "oversized" remainder $R^{*}$. Subsequent scaling of the reduced quotient by the short reciprocal yields an identity $N=Q^{*} D+R^{*}$. The oversized remainder $R^{*}$ may then be processed by a traditional integer division method (noting that essentially only one high radix digit need be determined) to augment $Q^{*}$ to obtain the integer quotient $Q$ and determine the final integer remainder $R$ satisfying $N=Q \times D+R$ with $0 \leq R \leq D-1$.

The general theory of our prescaled integer division method is outlined in Section 2. Our main result in Section 3 is a prescaled integer division algorithm employing a $k \times p$ digit rectangular aspect ratio multiplier that can be proven to determine the quotient and remainder of $2 p$ by $p$ digit division in $\left(\left\lceil\frac{p}{k-1}\right\rceil+3\right) k \times p$ digit multiplications.

In Section 4 we describe a version of prescaled integer division employing an adder for partial remainder computation where quotient digit selection in Booth radix 4 is available from the leading digit of the partial remainder. Both the divisor prescaling and the quotient postscaling operations are executable as 3-1 additions with one initial table lookup to determine the short divisor reciprocal value to be employed as the scale factor. 
Section 5 provides a conclusion and further directions for development of this procedure.

\section{Scaling Integer Division}

Consider integer division with the divisor $D$ being a $p$-digit base $\beta$ integer normalized to the range $\beta^{p-1} \leq$ $D \leq \beta^{p}-1$ and the dividend $N$ being a $2 p$-digit integer satisfying $0 \leq N \leq D \beta^{p}-1$. The result of integer division of $N$ by $D$ is an integer quotient $Q$ and an integer remainder $R$ which are uniquely determined by

$$
N=Q \times D+R, \text { with } 0 \leq R \leq D-1 .
$$

The bounds on $N$ and $D$ imply $0 \leq Q \leq \beta^{p}-1$ with both $Q$ and $R$ being $p$-digit base $\beta$ integers.

Digit serial division develops $Q$ by the dependent sequential steps

- next quotient digit selection

- partial remainder computation

Both of these steps can cost significant delay. When a rectangular aspect ratio $k \times p$ digit multiplier is available, the next quotient digit can be determined in the high radix $\beta^{k-1}$ by a $k \times p$ digit multiplication by a predetermined short reciprocal and rounding. The next partial remainder is then computable by a $k \times p$ digit multiplication. When only an adder is available to support digit serial division, the SRT algorithm employs table lookup for quotient digit selection and subtraction of a selected digit multiple of the multiplicand for remainder update.

A much simpler division process occurs when the divisor is a value very close to a power of the base. For the decimal divisor $D=100445568$, it is evident that the next quotient digit may be taken as the leading radix 100 digit of the partial remainder. Thus when the divisor is near a power of the base the steps of partial remainder computation and next quotient digit selection are effectively merged into a single operation, taking only about one half the time per high radix quotient digit generated.

Straightforward prescaled integer division scales a divisor and dividend by a predetermined integer scale factor $\bar{D}$ selected to generate a scaled divisor $D \bar{D}$ very close to a power of the base. Scaling both the integer divisor $N$ and dividend $D$ by $\bar{D}$ yields

$N \bar{D}=Q(D \bar{D})+R \bar{D}$ with $0 \leq R \bar{D} \leq D \bar{D}-\bar{D}$

Integer division of $N \bar{D}$ by $D \bar{D}$ provides a more efficient procedure to determine the identical integer quotient $Q$, but then concurrently provides a scaled remainder value $R \bar{D}$. This form of prescaled division is not applicable to integer division when both the integer quotient $Q$ and integer remainder $R$ must be determined.
We herein present an alternative prescaling algorithm that returns both the quotient $Q$ and remainder $R$ of integer division with nearly the same efficiency as a straightforward prescaling algorithm that would generate the same quotient with a scaled remainder.

Given $N$ and $D$ as in (1), we shall determine $Q$ and $R$ in terms of three intermediate integer parameters $q, r$, and $R^{*}$ that are defined as follows. Integer division of $Q$ by $\bar{D}$ uniquely specifies an integer reduced quotient $q$ and an integer reduced remainder $r$ by the equation

$$
Q=q \bar{D}+r, \quad 0 \leq r \leq \bar{D}-1 .
$$

Substituting equation (3) into equation (1) we obtain

$$
N=q D \bar{D}+(r D+R) .
$$

Letting $R^{*}=r D+R$, we obtain from the bounds $0 \leq r \leq \bar{D}-1$ and $0 \leq R \leq D-1$ that $0 \leq$ $R^{*} \leq \bar{D} \bar{D}-1$. Then $N=q \times(D \bar{D})+R^{*}$ with $0 \leq R^{*} \leq D \bar{D}-1$ means that the integers $q$ and $R^{*}$ can be determined by integer division of the (unscaled) dividend $N$ by the scaled divisor $D \bar{D}$.

We now wish to find $Q$ and $R$ from

$$
\begin{array}{r}
Q=q \bar{D}+r, \\
R=R^{*}-r D .
\end{array}
$$

Note that both $Q$ and $R$ can be found from a $k \times p$ digit multiply given that we can also determine $r$ having found $q$ and $R^{*}$ from the integer division of $N$ by $(D \bar{D})$. Observe that

$$
R^{*} \bar{D}=r(D \bar{D})+R \bar{D} .
$$

Since $r D \bar{D} \leq R^{*} \bar{D}<(r+1) D \bar{D}$ and since for an appropriately chosen $\bar{D}$ we can obtain $\frac{D \bar{D}}{\beta^{p+k-1}}=1+$ $\epsilon$ with $|\epsilon|<\frac{1}{\beta^{k-1}}$, the leading digit $r^{\prime}=\left\lfloor\frac{R^{*} \bar{D}}{\beta^{p+k-1}}\right\rfloor$ either equals $r$ or is very close to $r$. Using $r^{\prime}$ we may compute a trial quotient, by $q \bar{D}+r^{\prime}$ and trial remainder by $R^{*}-r^{\prime} D$, with a final small quotient and remainder adjustment as necessary if $R^{*}-r^{\prime} D$ is not in the range $[0, D-1]$. The process is formalized in the next section.

\section{Multiplicative Prescaled Integer Divi- sion}

For multiplicative prescaled integer division we assume support of a $k \times p$ digit base $\beta$ integer multiplier generating a $k+p$ digit product. Multiplicative prescaled division is particularly effective when the aspect ratio $p: k$ of the larger multiplicand size $p$ to the smaller multiplier size $k$ is in the range from $4: 1$ to $10: 1$. To obtain maximum benefit from the use of the $k \times p$ digit multiplier, it is critical that the scale factor 
used to prescale the divisor have both length and accuracy corresponding to the shorter size $k$ of the multiplier, allowing division by the prescaled divisor to be performed with digit selection in radix $\beta^{k-1}$.

For $1 \leq k<p$ with integer divisor $D$ satisfying $\beta^{p-1} \leq D<\beta^{p}$, let $\beta^{k-1} \leq \bar{D} \leq \beta^{k}-1$ and $0 \leq S \leq$ $D-1$ be determine by

$$
\beta^{k+p-1}=\bar{D} D-S \text {. }
$$

Here $\bar{D}=\left\lceil\frac{\beta^{k+p-1}}{D}\right\rceil$ is termed an integernormalized $k$-digit short reciprocal of the integer divisor $D$ and $S=\bar{D} D-\beta^{k+p-1}$ is the residue of $\bar{D} D$ modulo $\beta^{k+p-1}$. Equivalently, $\bar{D}$ is the quotient of $\beta^{k+p-1}$ divided by $D$ selected so as to have the negative remainder $-(D-1) \leq-S \leq 0$.

Prescaled integer division of the integer dividend $N$ by the integer divisor $D$ proceeds as follows

- The divisor $D$ is prescaled by a $k$-digit short reciprocal yielding the scaled divisor $D \bar{D}$.

- Integer division of $N$ by $D \bar{D}$ is performed with digit selection radix $\beta^{k-1}$, determining a reduced quotient, oversized remainder pair $q, R^{*}$ satisfying

$$
N=q(D \bar{D})+R^{*}, \text { with } 0 \leq R^{*} \leq D \bar{D}-1
$$

- The reduced quotient $q$ is scaled by $\bar{D}$ with the divisor "descaled" from $D \bar{D}$ to $D$ yielding the identity

$$
N=(q \bar{D}) D+R^{*}
$$

- A final quotient digit adjustment is performed to reduce the remainder from the oversized range $0 \leq$ $R^{*}<D \bar{D}-1$ to a remainder $R$ satisfying $0 \leq$ $R<D-1$.

The process is illustrated by the following numeric example.

Example: [Prescaled Integer Division]

Problem: For the $12 \times 6$ digit decimal integer division arguments

$$
\begin{array}{ll}
N=365748375204 & (12 \text { digit dividend) } \\
D=784731 & \text { (6 digit divisor) }
\end{array}
$$

find the 6 digit integer quotient $Q$ and non negative remainder $R$ with $0 \leq R \leq D-1$ such that

$$
N=Q \times D+\bar{R} \text {. }
$$

Note: We assume our multiplier is a $3 \times 6$ digit decimal multiplier, so $p=6, k=3$, and we pursue prescaled integer division in radix $10^{k-1}=100$.

Step 1: Determine the $k=3$ digit short reciprocal

$$
\bar{D}=\left\lceil\frac{10^{8}}{784731}\right\rceil=128 \text {. }
$$

Step 2: Determine the 9 digit scaled divisor and 6 digit residue

$$
\begin{aligned}
& D \times \bar{D}=784731 \times 128=100445568 \\
& S=445568
\end{aligned}
$$

Step 3: Perform digit serial division of the dividend by the the scaled divisor determining $\left\lceil\frac{p}{k-1}\right\rceil-1=2$ digits in the high radix 100. Note that digit selection is obtained directly from the leading digits of each partial remainder with the partial remainder computations comprising multiplications of the radix 100 digit by the 6 digit residue $S=445568$ in the $3 \times 6$ digit multiplier.

\begin{tabular}{ccc}
\multirow{3}{*}{100445568} & \multicolumn{3}{c}{3641.} & first digit selection \\
\cline { 2 - 2 } $36 \times 445568$ & $\frac{(36) 16040448}{\underline{41} 443304}$ & second digit selection \\
\multirow{3}{*}{$41 \times 445568$} & $\frac{(41) 18268288}{26062116}$
\end{tabular}

Step 4: Perform divisor descaling with quotient scaling,

$$
\begin{aligned}
365748375204 & =3641 \times 100445568+26062116 \\
(\text { divisor descaling:) } & =3641 \times(128 \times 784731)+26062116 \\
\text { (quotient scaling:) } & =(3641 \times 128) \times 784731+26062116 \\
& =466048 \times 784731+26062116
\end{aligned}
$$

Step 5: Reformat digit serial division and determine a low order digit incrementation by one step of the short reciprocal division procedure applied to the "oversized" remainder.

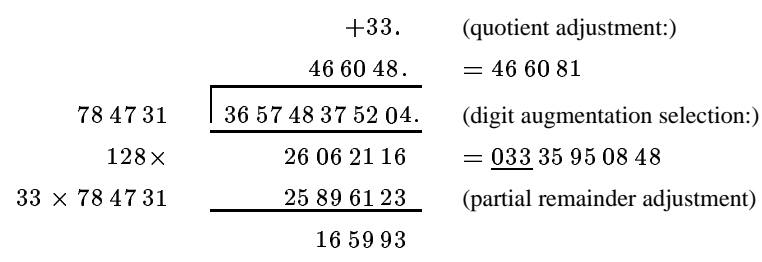

Step 6: Determine (confirm) positive remainder and provide quotient in non redundant form.

$$
\begin{aligned}
& Q=466081, \\
& R=165993, \quad 0 \leq R<784731 .
\end{aligned}
$$

The digit selection procedure for the first phase of Prescaled Integer Division utilized in Step 3 of the example is identical to digit selection in $p$ by $p$ digit prescaled division. It follows that $\left(\left\lceil\frac{p}{k-1}\right\rceil-1\right)$ high radix digits are determined at a cost of just one $k \times p$ digit multiplication per digit. The remainder $R^{*}=26062116$ in Step 5 in general satisfies $\left|R^{*}\right|<$ $\beta^{p+k-1}+S$, so multiplication by the short reciprocal determines a $p+2 k-1$ digit product with leading $k$ digit tuple magnitude no greater than the short reciprocal. Here we obtain $033 \leq 128$.

The digit selection multiplication in Step 5 can be performed as a $k \times p$ digit multiplication in the 
form $\bar{D} \times\left\lfloor\frac{R^{*}}{\beta^{k-1}}\right\rfloor=128 \times 260621=\underline{033} 359488$, with $R^{*} \bmod \beta^{k-1}$, (here being 16) then added to the remainder in Step 6. Since the augmented digit selected in Step 5 is in the range $[-\bar{D}, \bar{D}]$, the partial remainder adjustment can be supported by a $k \times p$ digit multiplication, with the resulting next partial remainder output in Step 5 possibly needing further adjustment to obtain absolute value less than the divisor $D$.

\section{Algorithm 1 (Prescaled Integer Division) \\ Stimulus:}

A high radix $\beta^{k-1}$ with $(k-1) \geq 2$, a precision $p$ with $p \geq k+1$, a p-digit integer divisor $D$ with $\beta^{p-1} \leq D \leq \beta^{p}-1$, a $2 p$-digit integer dividend $N$ with $0 \leq N \leq D \beta^{p}-1$.

\section{Response:}

An integer quotient $Q$ with $0 \leq Q \leq \beta^{p}-1$, an integer remainder $R$ with $0 \leq R \leq D-1$, where $Q, D$ satisfy $N=Q D+R$.

\section{Method:}

$Q:=0$

$\bar{D}:=\left\lceil\frac{\beta^{p+k-1}}{D}\right\rceil ;\{$ short reciprocal $\}$

$R=N ;\{$ initiate partial remainder $\}$

$S=D \bar{D}-\beta^{p+k-1} ;\{$ residue of scaled dividend $\}$

$n:=\lceil p /(k-1)\rceil ;\{$ number of high radix digits in $Q\}$

for $i:=n-1$ downto 1 do

(1) digit $:=R \operatorname{div} \beta^{i(k-1)+p} ;$ \{leading

digit selection $\}$

(2) $R:=R-$ digit $\times \beta^{i(k-1)+p} ;\{$ leading digit deletion $\}$

(3) $R:=R-$ digit $\times S \times \beta^{(i-1)(k-1)} ;\{$ partial remainder determination $\}$

(4) $Q:=Q \times \beta^{k-1}+$ digit; $\{$ quotient accumulation $\}$ od:

(5) $Q:=Q \times \bar{D} ;\{$ quotient scaling $\}$

(6) digit $:=(\bar{D} \times R) \operatorname{div} \beta^{(k-1)+p} ;\{$ digit augmentation selection $\}$

(7) $R:=R-$ digit $\times D$; \{partial remainder adjustment $\}$

(8) $Q:=Q+$ digit; \{quotient accumulation $\}$

(9) while $R<0$ do

$$
Q:=Q-1
$$$$
R:=R+D
$$

od;

(10) while $R \geq D$ do

$$
Q:=Q+1
$$$$
R:=R-D
$$

od;

Lemma 1 Algorithm 1 for Prescaled Integer Division performs $2 p$ by $p$ digit base $\beta$ integer division in the high radix $\beta^{k-1}$, with $k-1 \geq 2$, employing $\left(\left\lceil\frac{p}{k-1}\right)\right\rceil+$ 3) $k \times p$ digit multiplications.

Outline of Proof: A single $k \times p$ digit base $\beta$ multi- plication in Statement (3) determines both the next partial remainder and the next high radix quotient digit, yielding a total of $\left.\left(\left[\frac{p}{k-1}\right)\right]-1\right) k \times p$ digit multiplications in the for loop. The prescaling of the divisor determining $S$ and the postscaling of the quotient in Statement (5) contributes two more $k \times p$ digit multiplications. The remainder scaling in Statement (6) for low order digit augmentation selection and the partial remainder adjustment in Statement (7) can be performed by two more $k \times p$ digit multiplications for the total of $\left.\left(\left\lceil\frac{p}{k-1}\right)\right\rceil+3\right)$.

For correctness of Algorithm 1 note initially that $R=N<D \bar{D} \beta^{(n-1)(k-1)}$. Using induction for $i=n-1, n-2, \ldots, 1$, assume $|R|<D \bar{D} \beta^{i(k-1)}$. Consider the case $R \geq 0$. The rational $\frac{\beta^{p+k-1} R}{D \bar{D}}$ determines the digit string for the rest of the infinitely precise quotient, and since $\bar{D} \times R$ exceeds the value by less than one part in $\beta^{k-1}$, the high radix digit determined in Statement (1) is either identical to the leading digits of the string or is 1 unit larger. If identical, the next partial remainder $R$ satisfies $0 \leq R<D \bar{D} \beta^{(i-1)(k-1)}$, and if the high radix digit is 1 unit larger, the next partial remainder is negative with $0<|R|<D \bar{D} \beta^{(i-1)(k-1)}$. The case for $R<0$ follows by symmetry, so by induction we terminate the for loop with $N=Q^{\prime} \times D+R^{\prime}$, where $\left|R^{\prime}\right| \leq D \bar{D}-1$.

As noted in the paragraph before Algorithm 1, the final high radix digit determined in Statement (6) is in the range $[-\bar{D}, \bar{D}]$. Although this digit can exceed $\beta^{k-1}$, it does not exceed $\beta^{k}$ in magnitude and the remainder adjustment can be supported by a $k \times p$ digit multiplier, followed possibly by some further additive adjustments to obtain $0 \leq R \leq D-1$.

Note that determining a full $p \times p$ digit multiplication with a $k \times p$ digit multiplier employs $\left\lceil\frac{p}{k}\right\rceil p \times k$ digit multiplications. For $1<<k<<p$, it follows that the number of $p \times k$ digit multiplications employed for prescaled integer division to determine $Q, R$ satisfying $N=Q \times D+R$ is not much greater than the number of multiplies to compute $Q \times D+R$ to verify the answer. For example, employing a $12 \times 128$ bit multiplier for a $128 \times 128$ bit product utilizes 11 short-bylong multiplies, and finding the quotient and remainder by prescaled integer division as given by Algorithm 1 utilizes 15 short-by-long multiplies.

In summary the $2 p$ by $p$ digit prescaled integer division algorithm prescales only the initial divisor, and then postscales the resulting reduced quotient and oversized remainder of this modified division problem. The postscaling of the oversized remainder is employed to find a single "oversized" high radix digit to adjust the quotient and compute an appropriately bounded remainder. The foundation of the method with more details on determining the final high radix digit for quotient ad- 
justment is included in the following theorem.

Theorem 2 For $2 \leq k \leq(p-1)$, assume given a

- $p$-digit divisor $D$ with $\beta^{p-1} \leq D \leq \beta^{p-1}$,

- $2 p$-digit dividend $N$ with $0 \leq N \leq D \beta^{p-1}$,

- $k$-digit short reciprocal $\bar{D}$ and residual $S$ satisfying $\bar{D} D=\beta^{p+k-1}+S$ with $0 \leq S \leq D-1$,

- $[p-(k-1)]$-digit reduced quotient $q$ and oversized remainder $R^{*}$ satisfying $N=q \times(\bar{D} D)+$ $R^{*}$, with $0 \leq R^{*} \leq \bar{D} D-1$.

If $0 \leq R^{*} \leq D-1$, then the $p$-digit quotient $Q=q \times \bar{D}$ and remainder $R=R^{*}$ satisfies $N=Q D+R$ with $0 \leq R \leq D-1$

Else if $D \leq R^{*} \leq \bar{D} D-1$, then the quotient $Q$ and remainder $R$ given by

$$
\begin{aligned}
& Q=q \times \bar{D}+\left\lfloor\frac{R^{*} \times \bar{D}-S}{\beta^{p+k-1}}\right\rfloor, \\
& R=R^{*}-\left(\left\lfloor\frac{R^{*} \times \bar{D}-S}{\beta^{p+k-1}}\right\rfloor \times D\right), \\
& \text { satisfies } N=Q D+R \text { with }-D \leq R \leq D-1 .
\end{aligned}
$$

\section{Additive Prescaled Integer Division}

Our additive prescaled integer division algorithm will use a slightly different procedure for determining the postscaled quotient and corresponding remainder.

For the radix 4 we will assume our short reciprocal $\bar{D}$ is a 3 -digit radix 4 integer. We divide $N$ by the scaled divisor $D \bar{D}$ so as to obtain 3 fractional digits giving

$$
N=\frac{Q^{\prime}}{64}(D \bar{D})+\frac{R^{\prime}}{64}
$$

Letting $\frac{Q^{\prime} \bar{D}}{64}=Q^{\prime \prime}+\frac{j}{64}$ we obtain

$$
N=Q^{\prime \prime} D+\frac{j D+R^{\prime}}{64}
$$

Now $j D+R^{\prime}$ must be divisible by 64 , yielding an integer $R^{\prime \prime}=\frac{j D+R^{\prime}}{64}$ where $\left|R^{\prime \prime}\right|<2 D$. Thus $N=Q^{\prime \prime} D+R^{\prime \prime}$ where an augmentation of $Q^{\prime \prime}$ by at most a value from $\{-1,1,2\}$ yields $N=Q D+R$ with $0 \leq R \leq D-1$. Division radix 4 requires generation of $\left\lfloor\frac{p+1}{2}\right\rfloor$ digits in the Booth radix 4 quotient representation with digits $\{-2,-1,0,1,2\}$, which for $p=64$ is 33 digits. Since the number of quotient digits is relatively large, the generation of an additional 3 fractional digits with quotient and remainder adjustment by adding a fractional part times $D$ back into the remainder does not significantly increase the number of additive steps on a relative basis.

Our additive algorithm assumes $\bar{D}$ (normalized to $(1,2])$ is chosen so that $1 \leq D \bar{D}<1 \frac{1}{6}$ for a radix 4 divisor $D$ normalized to $\frac{1}{2} \leq D<1$, the above Table shows that a 3 digit $\bar{D}$ may be chosen so that $1 \leq D \bar{D}<1 \frac{1}{6}$. Our partial remainder computation

\begin{tabular}{|c|c|c|}
\hline $\begin{array}{c}\text { Divisor } \\
\text { Interval }\end{array}$ & $\begin{array}{c}\text { Short Reciprocal } \\
\bar{D}\end{array}$ & $\begin{array}{c}\text { Scaled Interval } \\
D \bar{D}\end{array}$ \\
\hline \hline$\left[\frac{1}{2}, \frac{4}{7}\right)$ & 2.00 & {$\left[1, \frac{8}{7}\right)$} \\
\hline$\left(\frac{4}{7}, \frac{2}{3}\right)$ & $\frac{7}{4}=2 . \overline{1} 0$ & $\left(1, \frac{7}{6}\right)$ \\
\hline$\left(\frac{2}{3}, \frac{3}{4}\right)$ & $\frac{4}{3}=1.20$ & $\left(1, \frac{9}{8}\right)$ \\
\hline$\left[\frac{3}{4}, \frac{4}{5}\right)$ & $\frac{11}{8}=1.12$ & $\left(\frac{33}{32}, \frac{11}{10}\right)$ \\
\hline$\left(\frac{4}{5}, \frac{8}{9}\right)$ & $\frac{4}{5}=1.10$ & $\left(1, \frac{10}{9}\right)$ \\
\hline$\left(\frac{8}{9}, 1\right)$ & $\frac{9}{8}=1.02$ & $\left(1, \frac{9}{8}\right)$ \\
\hline
\end{tabular}

is performed so that when the leading digit of the partial remainder is 1 or 2 , then all subsequent digits are in the set $\{-1,0,1,2\}$ constituting a fractional part in the range $\left(-\frac{1}{3}, \frac{2}{3}\right)$. Similarly when the partial remainder is -1 or -2 , all subsequent digits are in the set $\{-2,-1,0,1\}$ constituting a fractional part in the range $\left(-\frac{2}{3}, \frac{1}{3}\right)$. This provides for partial remainder computation of $R:=4(R-d D)$, where $d \in\{-2,-1,0,1,2\}$ is chosen to maintain the condition that $(R-d D)$ is in the range $\left(-\frac{2}{3}, \frac{2}{3}\right)$. The following example illustrates this additive integer prescaled division procedure for integer division of an 8 digit radix 4 dividend by a 4 digit radix 4 divisor.

[Additive Prescaled Integer Division]

Problem: For the $8 \times 4$ digit radix 4 integer division arguments

$$
\begin{array}{ll}
N=13222012_{4} & (8 \text { digit dividend }) \\
D=2211_{4} & (4 \text { digit divisor })
\end{array}
$$

find the 4 digit integer quotient $Q$ and remainder $R$ with $0 \leq R \leq D-1$ such that

$$
N=Q \times D+R
$$

We assume our adder is able to perform signed digit summations and compress the remainder to either the digit set $\{-2,-1,0,1\}$ or $\{-1,0,1,2\}$

Step 1: Determine the three digit short reciprocal according to Table 1.

$\bar{D}=2 \overline{1} 0_{4}$ since $\frac{2211_{4}}{4^{4}}$ is in the interval $\left(\frac{4}{7}, \frac{2}{3}\right)$.

Step 2: Determine the 7 digit scaled divisor $D \bar{D}$

$D \times \bar{D}=2211 \times 2 \overline{1} 0=1102200_{4}=10201 \overline{1} 0_{4}$

Step 3: Perform digit serial division of the dividend by the the scaled divisor determining 5 quotient digits including 3 fractional digits radix 4 . Note that digit selection is obtained from the leading digit of the new partial remainder. If the leading digit is not zero and the second digit has an opposite sign or is zero, the bias of the digit set is changed from $\{-2,-1,0,1\}$ to $\{-1,0,1,2\}$ or from $\{-1,0,1,2\}$ to $\{-2,-1,0,1\}$ in compressing the next partial remainder. The positive dividend becomes the initial partial remainder with compression to $\{-1,0,1,2\}$. The leading two digits of each partial remainder are underlined to indicate the control. 
$2 \overline{1} . \overline{1} 1 \overline{2}$

$$
\begin{aligned}
& 1 0 2 0 1 \overline { 1 } 0 \longdiv { 2 \overline { 1 } 2 2 2 0 1 2 . 0 0 0 } \\
& 21002 \overline{2} \\
& \begin{array}{c}
\frac{11}{\overline{1} 0} \overline{2} 0 \overline{1} 120 \\
\frac{10}{\overline{1} 0 \overline{2} 0 \overline{1} 10} \\
\frac{1111100}{10201 \overline{1} 0} \\
\frac{2110100}{\overline{2} \overline{1} 00 \overline{2} 20} \\
\hline 210220
\end{array} \\
& \in\{-1,0,1,2\} \\
& \in\{-2,-1,0,1\} \\
& \in\{-2,-1,0,1\} \\
& \in\{-1,0,1,2\} \\
& \in\{-2,-1,0,1\} \\
& \in\{-1,0,1,2\}
\end{aligned}
$$

Step 4: Additively perform divisor descaling with quotient scaling,

$$
\begin{aligned}
2 \overline{1} 22012 & =2 \overline{1} . \overline{1} 1 \overline{2} \times 10201 \overline{1} 0+210.22 \\
& =2 \overline{1} . \overline{1} 1 \overline{2} \times(2 \overline{1} 0 \times 2211)+210.22 \\
& =(2 \overline{1} . \overline{1} 1 \overline{2} \times 2 \overline{1} 0) \times 2211+210.22 \\
& =(1 \overline{1} 0 \overline{1} 2 . \overline{1} 2) \times 2211+210.22
\end{aligned}
$$

Step 5: Add fractional quotient digits times divisor into remainder.

$$
\begin{aligned}
R= & . \overline{1} 2 \times 2211+210.22 \\
& =\overline{1} \overline{1} \overline{1} .12+210.22 \\
& =100
\end{aligned}
$$

Step 6: Determine (confirm) positive remainder and provide quotient in non redundant form.

$$
\begin{aligned}
& Q=2332_{4}, \\
& R=100_{4}, \quad 0 \leq R \leq D-1 .
\end{aligned}
$$

Observation 2 The additive Prescaled Integer Division procedure as described in conjunction with the example yields a quotient $Q$ and remainder $0 \leq R \leq D-1$ satisfying $N=Q D+R$.

The procedure described may be improved so that the partial remainder need not be fully compressed to obtain the next quotient digit. It can be shown that a short reciprocal table can be determined requiring no more than 3 digits in $\bar{D}$ where the scaled divisor $D \bar{D}$ is restricted to the narrower range $\left[1,1 \frac{1}{15}\right)$. This allows partial compression of the redundant partial remainder where effectively only a couple leading digits of the partial remainder need be compressed to obtain a quotient digit that will preserve the sufficiently compressed range of the redundant partial remainder. The determination of appropriate reciprocal values is aided by the methodology in [8]. The possibility of using only a limited number of leading digits of the redundant remainder to determine the next quotient digit follows from the methodology in [5].

By using higher precision table lookup of the reciprocal to bound the size of $D \bar{D}$ in a much smaller neighborhood of unity, it is possible with partial compression of the remainder to perform additive integer prescaled division with higher radices such as radix 8 or 16 . Employing efficient partial compression techniques such as described in [2, 4, 6, 7] might allow implementations where a radix 8 or 16 digit could be determined each cycle.

\section{Conclusion}

We have demonstrated a new divisor-prescaling quotient-postscaling digit serial division algorithm that can be applied to integer division. The procedure allows each quotient to be computed at a rate of one high radix quotient digit per $k \times p$ digit multiplication for the multiplicative version and one high radix quotient digit per partially compressed addition for the additive version, with the remainder available at an additional cost essentially independent of the number of digits generated in the quotient. Fine tuning of the additive version and some implementation studies of the algorithms are planned to determine the overall feasibility of these integer division algorithms compared to current implementations of existing integer division methods.

\section{References}

[1] D. E. Atkins, "Higher-Radix-Division Using Estimates of the Divisor and Partial Remainders" IEEE Transactions on Computers, vol. 17, no. 10, pp. 925934, 1968.

[2] M. Daumas and D. W. Matula, "Further reducing the Redundancy of a Notation Over a Minimally Redundant Digit Set", J. VLSI, vol. 33, pp. 7-18, 2003 (see also: M. Daumas and D. W. Matula, "Recoders for Partial Compression and Rounding", Research Report 97-01 Laboratoire de l'Informatique du Parallelisme, Lyon, France, 1997).

[3] M. D. Ercegovac and T. Lang, Division and Square Root, Norwell, Mass.: Kluwer Academic, 1994.

[4] P. Kornerup, "Digit-Set Conversion: Generalizations and Applications" IEEE Transactions on Computers, vol. 43, no. 5, pp. 622-629, 1994.

[5] L. A. Montalvo, K. K. Parhi, and A. Guyot, "New Svoboda-Tung Division", IEEE Transactions on Computers, vol. 47, no. 9, pp. 1014-1020, 1998.

[6] A. N. Nielsen, D. W. Matula, C. N. Lyu and G. Even, "An IEEE Compliant Floating Point Adder that Conforms with the Pipelined Packet Forwarding Paradigm", IEEE Transactions on Computers, vol. 49, no. 4, pp. 33-47, 2000.

[7] P.-M. Seidel "High Speed Redundant Reciprocal Approximation" 3rd Real Numbers and Computers Conference, Paris, France, pp. 219-229, 1998.

[8] D. Das Sarma and D. W. Matula, "Measuring the Accuracy of ROM Reciprocal Tables.", IEEE Transactions on Computers, vol. 43, no. 8, pp. 932-940, 1994.

[9] A. Svoboda,"An Algorithm for Division", Information Processing Machines, no. 9, pp. 25-32, 1963. 